\title{
Association between response to triptans and response to erenumab: real-life data
}

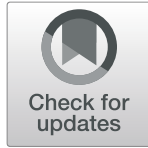

\author{
Ilaria Frattale ${ }^{1}$, Valeria Caponnetto ${ }^{1}$, Alfonsina Casalena ${ }^{2}$, Maurizio Assetta ${ }^{2}$, Maurizio Maddestra ${ }^{3}$, Fabio Marzoli ${ }^{3}$, \\ Giannapia Affaitati ${ }^{4}$, Maria Adele Giamberardino ${ }^{4}$, Stefano Viola ${ }^{5}$, Amleto Gabriele ${ }^{6}$, Francesca Pistoia ${ }^{1,7}$, \\ Davide Cerone ${ }^{7}$, Carmine Marini ${ }^{8}$, Simona Sacco ${ }^{1 *}$ (i) and Raffaele Ornello ${ }^{1}$
}

\begin{abstract}
Background: Triptans and erenumab are both migraine-specific agents acting on the calcitonin gene-related peptide pathway. Therefore, response to triptans might be associated with response to erenumab.

Main body: In our study, consecutive patients referring to the Headache Centers of the Abruzzo region from January 2019 to March 2020 and treated with erenumab were interviewed about past use and efficacy of triptans. Triptan users were classified as 'triptan responders' if they were headache-free $2 \mathrm{~h}$ after treating $\geq 3$ migraine attacks with $\geq 1$ triptan. We considered patients as 'erenumab responders', if they had a $\geq 50 \%$ mean reduction in monthly migraine days between the 4th and the 6th month from treatment start compared with baseline. Of 91 triptan users, 73 (80.2\%) were triptan responders and 58 (63.7\%) were erenumab responders. The odds ratio of being erenumab responder was $3.64(95 \% \mathrm{Cl}, 1.25-10.64)$ for triptan users as compared to non-users. $(P=0.014)$. Besides, starting erenumab improved triptan response in both erenumab responders and non-responders.
\end{abstract}

Conclusions: Our data of an association between response to triptans and response to erenumab can be useful for patient advice and to improve the understanding of migraine pathophysiology and treatment.

Keywords: Triptans, Erenumab, Migraine treatment, CGRP

\section{Background}

Migraine affects $14.4 \%$ of adults worldwide [1]. Despite the high burden of migraine, preventive treatments were not disease-specific until the advent of monoclonal antibodies targeting the calcitonin gene-related peptide (CGRP) pathway [2, 3]. During a migraine attack, the activation of the trigeminovascular system has a key role, leading to CGRP release at the trigeminal endings; this leads in turn to vasodilation of the intracranial arteries, modulates neuronal excitability through the facilitation of pain transmission, and activates neurogenic inflammation $[3,4]$. Monoclonal antibodies targeting CGRP or

\footnotetext{
* Correspondence: simona.sacco@univaq.it

${ }^{1}$ Neuroscience Section, Department of Applied Clinical Sciences and Biotechnology, University of L'Aquila, Via Vetoio 1, 67100 L'Aquila, Italy Full list of author information is available at the end of the article
}

its receptor lead to modulation of pain transmission and reduction in both peripheral and central sensitization by removing the excess of released CGRP (anti-CGRP antibodies) or the block of the ligand from binding the CGRP receptor (anti-CGRP receptor antibodies) [4].

While the development of migraine-specific preventive treatments is recent, acute treatments that are specific for migraine are available since the 1990s. Triptans are agonists of the 5-hydroxytryptamine (5-HT) receptors 5HT1B, 5-HT1D and 5-HT1F [5]. 5-HT1B and 5-HT1D receptors are localized in trigeminal ganglia and trigeminal nerves; 5-HT1D receptor are detected in trigeminal nerves projecting peripherally to the dural vasculature to inhibit activated trigeminal nerves and prevent vasoactive neuropeptide release, and centrally to the brainstrem trigeminal nuclei to interrupt pain signal transmission [6]. At the 
peripheral level, triptans constrict extracerebral blood vessels and reduce trigeminal sensory nerve activation, thus ultimately inhibiting vasoactive peptide release including substance P and CGRP [7]. Therefore, triptans indirectly share their target with monoclonal antibodies targeting the CGRP pathway [8]. Hence, it can be speculated that migraineurs whose attacks respond to triptans might also have a favorable response to the new migraine-specific preventive treatments.

In the present real-life study, we assessed 1) whether previous response to triptans predicts the subsequent efficacy of erenumab; 2) whether the loss of efficacy wear-off - of triptans over time predicts erenumab ineffectiveness, and 3) whether erenumab treatment improves the efficacy of triptans.

\section{Methods}

This is an ancillary study from a real-life observational study on patients treated with erenumab [9]. The study was approved by the Internal Review Board of the University of L'Aquila with protocol number 44/2019. The study database is available from the Corresponding Author upon reasonable request.

Our study included patients aged 18 to 65 years and consecutively treated with erenumab from January 2019 to March 2020 in the Headache Centers of Avezzano, L'Aquila, Sulmona, Teramo, Chieti, Lanciano, and Vasto. All patients had a diagnosis of migraine with or without aura according with International Classification of Headache Disorders (ICHD) criteria [10]. According to clinical indications for erenumab, we included patients with chronic or high-frequency ( $\geq 8$ monthly headache days) episodic migraine. For each patient we recorded sex, age, and migraine characteristics.

We collected information on the use of triptans by means of a structured data collection form administered via telephone interview. The data collection form was designed by consensus among the study participants according to their clinical experience. Patients were asked about past use of the six triptans available in Italy, namely almotriptan, eletriptan, frovatriptan, rizatriptan, sumatriptan, and zolmitriptan, at any time before starting erenumab treatment. Information was checked with medical chart review where available. We only included patients who signed a written informed consent to participate in an observational study on monoclonal antibodies targeting the CGRP pathway; oral additional consent prior to the questionnaire was deemed sufficient to participate in the present study. Patients were considered not available on the phone if not answering after three phone call attempts.

Patients who reported use of at least one triptan for at least three migraine attacks were considered triptan users. Triptan users were classified as 'triptan responders' if they were headache-free within $2 \mathrm{~h}$ after treating at least three migraine attacks with one triptan [11]; the remaining patients were classified as 'triptan non-responders'. Triptan responders were also asked about a decreased response to triptans over time, configuring the 'wear-off' phenomenon. Those same patients were also asked whether the efficacy of triptans improved after starting treatment with erenumab.

Regarding erenumab treatment, patients were classified as 'erenumab responders' if reporting a mean $\geq 50 \%$ reduction in monthly migraine days from baseline (i.e. the three months preceding the start of erenumab treatment) to month 4-6 of treatment, in accordance with a secondary end-point of the STRIVE trial [12]; the remaining patients were classified as 'erenumab nonresponders'.

\section{Statistical analysis}

We included in primary analyses all triptan users. We reported descriptive statistics by using numbers and proportions or means and standard deviations (SDs) as appropriate. We reported the odds ratios (ORs) and 95\% confidence intervals (CIs) of the association between triptan response and erenumab response by means of the chi squared statistics. According to the calculations made with G*Power software [13], we estimated that performing a chi squared analysis with a total sample size of $n=88$ would be sufficient to detect a medium (f $=0.3$ ) effect size between two groups with a $P$ value < 0.05 and $80 \%$ power. We planned subgroup analyses on 1) patients using triptans during the three months before starting erenumab treatment and 2) patients who continued using triptans during erenumab treatment. We used Microsoft Excel and SPSS version 20 to perform the analyses.

\section{Results}

During the study period, 140 patients were treated with erenumab for at least 6 months; 105 of them $(75.0 \%)$ answered to the data collection form, while 35 (25.0\%) were not available on the phone. Among the 35 patients not answering to the data collection form, 16 (45.7\%) were erenumab responders and 19 (54.3\%) erenumab non-responders. Ninety-one (86.6\%) of the 105 patients responding to the data collection form were considered triptan users and included in the study (Fig. 1). Regarding erenumab, $58(63.7 \%)$ of the included patients were erenumab responders and $33(32.3 \%)$ were erenumab non responders; regarding triptans, $73(80.2 \%)$ were triptan responders and 18 (19.8\%) triptan non-responders. There were no major differences in baseline characteristics between erenumab responders and non-responders (Table 1); the only significant difference regarded triptan use. In fact, among erenumab responders, 51 (87.9\%) 
Table 1 Comparisons between erenumab responders and non-responders in the 91 triptan users

\begin{tabular}{|c|c|c|c|}
\hline & Erenumab responders $(n=58)$ & Erenumab non-responders $(n=33)$ & $P$ value \\
\hline \multicolumn{4}{|l|}{$N, \%$} \\
\hline Female & $49(84.5)$ & $30(90.9)$ & 0.384 \\
\hline Chronic Migraine & $53(91.4)$ & $29(87.9)$ & 0.591 \\
\hline Medication overuse & $38(65.5)$ & $23(69.7)$ & 0.683 \\
\hline Aura & $21(36.2)$ & $10(30.3)$ & 0.568 \\
\hline Allodynia & $33(56.9)$ & $15(45.5)$ & 0.293 \\
\hline Preventive treatment failures & & & 0.464 \\
\hline $2-4$ & $36(62.1)$ & $23(69.7)$ & \\
\hline$>4$ & $22(37.9)$ & $10(30.3)$ & \\
\hline Triptan responders & $51(87.9)$ & $22(66.7)$ & 0.014 \\
\hline \multicolumn{4}{|l|}{ Mean $\pm S D$} \\
\hline Age & $46.6 \pm 9.5$ & $46.6 \pm 10.9$ & 0.926 \\
\hline Migraine duration, years & $25.0 \pm 11.2$ & $29.3 \pm 12.3$ & 0.143 \\
\hline Monthly headache days & $21.8 \pm 7.9$ & $18.3 \pm 9.4$ & 0.084 \\
\hline Monthly migraine days & $18.2 \pm 7.1$ & $17.8 \pm 9.3$ & 0.827 \\
\hline Monthly medication days & $18.2 \pm 8.7$ & $18.6 \pm 8.2$ & 0.833 \\
\hline Mean headache intensity & $7.8 \pm 1.8$ & $7.7 \pm 1.7$ & 0.792 \\
\hline
\end{tabular}

were triptan responders while among erenumab nonresponders, 22 (66.7\%) were triptan responders. The OR of being erenumab responder was 3.64 (95\% CI, 1.2510.64) for triptan responders as compared to nonresponders $(P=0.014$; Fig. 2 -a).

Forty-six triptan users (50.5\%) reported triptan wearoff. Among erenumab responders, 28 (48.3\%) had triptan wear-off, while among erenumab non-responders 18 (54.5\%) had triptan wear-off. The OR of being erenumab responder was 0.78 (95\% CI, 0.33-1.83) for patients reporting triptan wear-off as compared to those not reporting triptan wear-off ( $P=0.565$; Fig. 2 -a).

Sixty-five patients $(71.4 \%)$ were triptan users during the three months preceding erenumab treatment; 60 (92.3\%) of them were triptan responders, while the remaining 5 (7.7\%) were triptan non-responders. Due to low numbers, we did not perform a subgroup analysis on those patients.

After starting erenumab treatment, 52 patients (57.1\%) continued using triptans; 36 of them (69.2\%) were

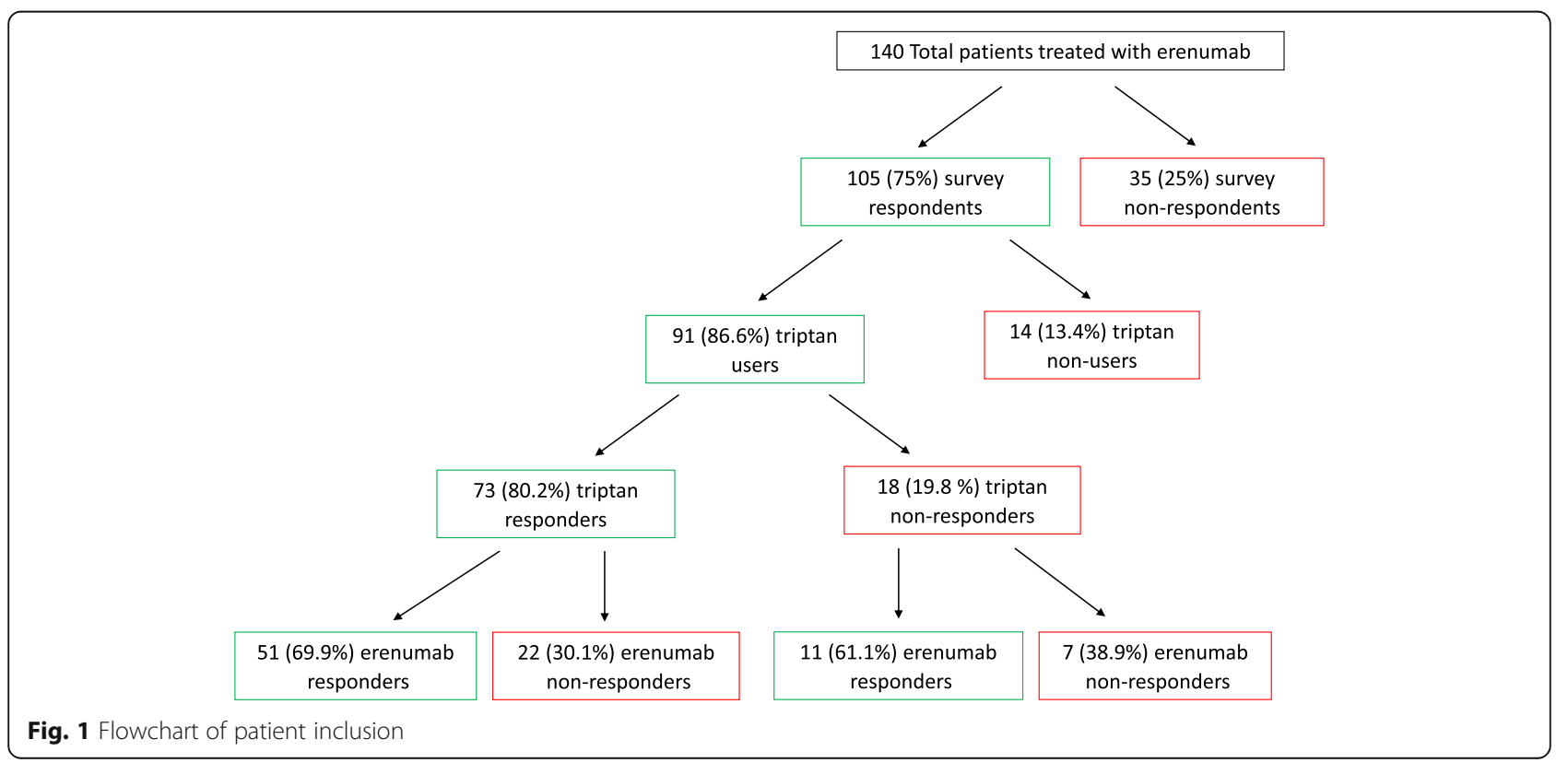




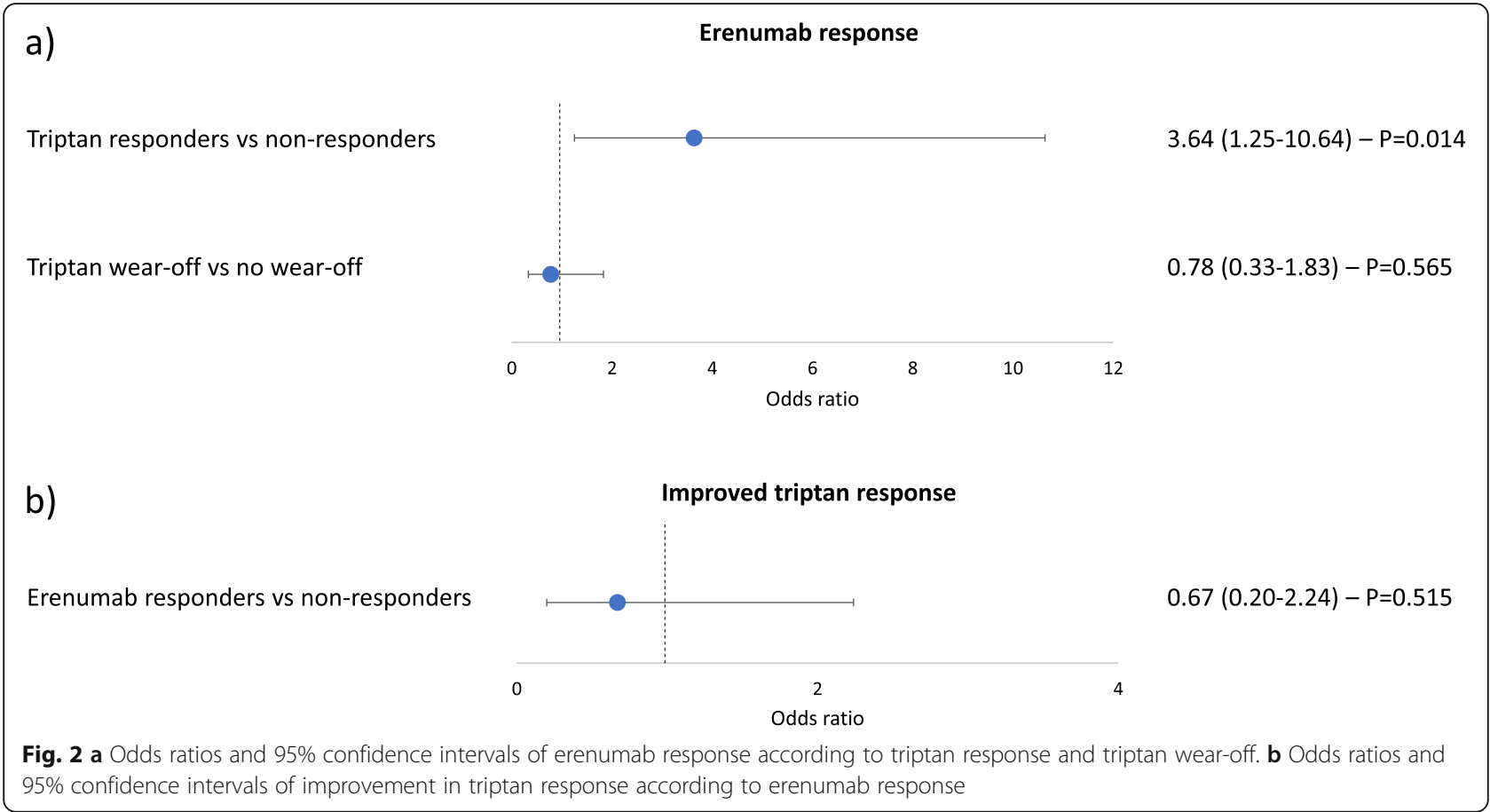

erenumab responders and $16(30.8 \%)$ erenumab nonresponders. Twenty-nine $(55.8 \%)$ patients in the overall group, 19 (52.8\%) erenumab responders and 10 (62.5\%) erenumab non-responders reported an improvement in triptan effectiveness. The proportion of patients reporting an improvement in triptan effectiveness was similar in erenumab responders and erenumab non-responders (52.8\% vs $62.5 \%$; OR 0.67; 95\% CI, $0.20-2.24 ; P=0.265$; Fig. 2-b).

\section{Discussion}

In our study, patients showing a favorable response at any time to at least one triptan had a higher probability to be responders to erenumab compared with those not responding to triptans. This information is important as it may improve our understanding of migraine pathophysiology and treatment; it could also be used in clinical practice to advise patients about their chances of response to erenumab treatment. However, previous response to triptans alone should not represent a strict criterion to select patients for erenumab treatment, because many triptan non-responders were erenumab responders.

To our knowledge, this is the first study primarily addressing the association between the response to triptans and that to monoclonal antibodies targeting the CGRP pathway. A previous real-life study found a trend toward better response to erenumab in triptan responders compared with non-responders [14], being however underpowered to draw definite conclusions. The remaining available real-life studies on the safety and efficacy of erenumab [15-20] did not assess triptan response. Notably, the proportion of patients responding to erenumab in our cohort was higher than in randomized clinical trials $[12,21]$, while it was comparable to other real-life studies [14-16]. Our proportion of triptan users was also higher than those of trials $[12,21]$, while no comparable data are available for real-life studies.

The findings of our study can be explained considering what we know about migraine pathophysiology. A common action on the trigeminovascular system [8] might explain the association between response to triptans and response to erenumab. A previous study found that patients responding to rizatriptan had higher jugular blood levels of CGRP during migraine episodes compared with patients not responding to rizatriptan; besides, patients responding to rizatriptan had a steep decrease in CGRP after the administration of rizatriptan, which was not found in non-responders [11]. In triptan non-responders, pain neurotransmitters different from CGRP might be important in the generation of migraine; thus, triptan nonresponders might be less responsive to CGRP-targeted treatments. It is important to note that while erenumab was designed as a CGRP receptor blocker, the action of triptans on CGRP is indirect.

Our finding of an association between response to triptans and response to a migraine-specific preventative is in line with a previous report which found an association between response to triptans and response to onabotulinumtoxin A [22]. However, the association found by this early study was not confirmed in a further report [23]. The rationale for those studies was that 
onabotulinumtoxin A acts on the trigeminovascular system as well as triptans [8]. Onabotulinumtoxin A acts on peripheral nerves terminal to interfere with specific events in the synaptic vesicle cycle. These vesicles contain small molecules like acetylcholine and glutamate or vasodilatory neuropeptides including pituitary adenylate cyclase activating peptide 38 (PACAP 38), Substance P, and CGRP. Thus, onabotulinumtoxin A injection inhibits the release of these neuropeptides from primary sensory first order neurons in dorsal root and trigeminal ganglia [24]. During a migraine attack, the headache phase depends on activity in unmyelinated $\mathrm{C}$ - and thinly myelinated $\mathrm{A} \delta$-fibers in the dura. Onabotulinumtoxin $\mathrm{A}$ appears to selectively inhibit the activation and sensitization of the unmyelinated $C$ but not thinly myelinated $A \delta$ fibers [25]. Preclinical evidence suggests that monoclonal antibodies acting on CGRP inhibits $A \delta$ but not $C$ type neurons in the trigeminal ganglion [26], therefore having an activity complementary to that of onabotulintoxinA. Taking all the evidence together, monoclonal antibodies acting on the CGRP pathway, triptans, and onabotulinumtoxin A all are involved in inhibition of the release of CGRP, but at the same time they also act on different pathways implicated in the pathogenesis of migraine.

Data about the improvement in response to acute medication are not reported by randomized clinical trials, despite being relevant in clinical practice as an additional efficacy outcome of migraine preventive medication. Improving responsiveness to acute medication is a goal of migraine prevention [27] and might be an additional parameter to test the efficacy of preventative drugs. In our study, after starting erenumab, more than half of patients reported an improvement in their response to triptans. This favorable effect was appreciated not only in erenumab responders but also in erenumab non-responders; on the contrary, among patients treated with onabotulinumtoxin A only responders reported an improved response to triptans [23]. The improved response to triptans found in patients treated with erenumab can be explained by synergy, as erenumab blocks the CGRP receptor, while the target of triptans is not the CGRP receptor itself. We cannot exclude that the improved response to triptans might also be explained by an overall improved response to acute medication, including triptans and non-steroidal anti-inflammatory drugs, in patients treated with a migraine preventative.

Our data are preliminary and should be taken with caution, as they come from a real-life, non-randomized study. Our study is also limited by a small sample size, allowing reliable univariate comparisons but not multivariate adjustments. The limited number of patients treated with triptans during the three months before erenumab treatment and after starting the treatment also limited the possibility of performing subgroup analyses. Despite the high rate of response to the questionnaire (75\%), ensuring the reliability of our sample, the retrospective recall of information through telephone interview is prone to recall bias. Moreover, due to the limited number of patients we could not address differences according to the different triptans. Lastly, our data only refer to patients treated with erenumab, a CGRP receptor antagonist, and are therefore not generalizable to monoclonal antibodies targeting the CGRP molecule.

\section{Conclusion}

According to our real-life data, patients reporting response to at least one triptan have a higher likelihood to respond to erenumab treatment compared with triptan non-responders. This information is relevant to improve our understanding of migraine and its treatments and to predict the efficacy of migraine-specific preventatives.

\section{Abbreviations}

5-HT: 5-hydroxytriptamine; CGRP: Calcitonin gene-related peptide;

ICHD: International Classification of Headache Disorders; STRIVE: Study to Evaluate the Efficacy and Safety of Erenumab (AMG 334) in Migraine Prevention

\section{Acknowledgments}

The Authors thank the study patients for their kind cooperation.

\section{Authors' contributions}

IF drafted the initial manuscript and revised it for intellectual content. SS and RO conceived the study, collected clinical data, and revised the manuscript. $\mathrm{Al}$ the remaining Authors collected the data and revised the manuscript for intellectual content. The authors read and approved the final manuscript.

\section{Funding}

the publication fee for the present paper was unconditionally granted by Novartis Farma S.r.l.

\section{Availability of data and materials}

anonymized data operated or analyzed during this study are available from the Authors upon reasonable request.

Ethics approval and consent to participate

the study was approved by the Internal Review Board of the University of L'Aquila (Italy) and patients gave written informed consent according to the Declaration of Helsinki.

\section{Consent for publication}

not applicable.

\section{Competing interests}

$\mathrm{RO}$ declares financial and non-financial relationships with Eli Lilly and Novartis, non-financial relationships with Allergan, and Teva; SS had a financial relationship (lecturer or member of advisory board) with Abbott, Allergan, Novartis, Teva, and Eli Lilly; GA has received funds for congress participation from Innovet Italia Srl, Epitech Group and Lusofarmaco; MAG received funds for congress participation from IBSA; AC, IF, AG, MA, MM, FM, SV, DC, CM, and FP declare no competing interests.

\section{Author details}

${ }^{1}$ Neuroscience Section, Department of Applied Clinical Sciences and Biotechnology, University of L'Aquila, Via Vetoio 1, 67100 L'Aquila, Italy. ²Department of Neurology, 'G. Mazzini' Hospital, Teramo, Italy. ${ }^{3}$ Department of Neurology, 'F. Renzetti' Hospital, Lanciano, Italy. ${ }^{4}$ Department of Medicine and Science of Aging, 'G. D'Annunzio' University, Chieti, Italy. ${ }^{5}$ Department of Neurology, 'S. Pio da Pietrelcina' Hospital, Vasto, Italy. ${ }^{6}$ Neurology Service, 
'SS.Annunziata' Hospital, Sulmona, Italy. ${ }^{7}$ Department of Neurology, 'S. Salvatore' Hospital, L'Aquila, Italy. ${ }^{8}$ Department of Internal Medicine, Public Health, Life and Environmental Sciences, University of L'Aquila, L'Aquila, Italy.

Received: 24 October 2020 Accepted: 12 December 2020

Published online: 06 January 2021

\section{References}

1. Global Burden of Disease 2016 Collaborators (2018) Global, regional, and national burden of migraine and tension-type headache, 1990-2016: a systematic analysis for the Global Burden of Disease Study 2016. Lancet Neurol 11:954-976. https://doi.org/10.1016/S1474-4422(18)30322-3

2. De Matteis E, Guglielmetti M, Ornello R, Spuntarelli V, Martelletti P, Sacco S (2020) Targeting CGRP for migraine treatment: mechanisms, antibodies, small molecules, perspectives. Expert Rev Neurother:1-15. https://doi.org/10. 1080/14737175.2020.1772758

3. Tiseo C, Ornello R, Pistoia F, Sacco S (2019) How to integrate monoclonal antibodies targeting the calcitonin gene-related peptide or its receptor in daily clinical practice. J Headache Pain 20(1):49. https://doi.org/10.1186/ s10194-019-1000-5

4. Edvinsson $L$ (2017) The Trigeminovascular pathway: role of CGRP and CGRP receptors in migraine. Headache. 57(Suppl 2):47-55. https://doi.org/10.1111/ head.13081

5. Ahn AH, Basbaum Al (2005) Where do triptans act in the treatment of migraine? Pain. 115(1-2):1-4. https://doi.org/10.1016/j.pain.2005.03.008

6. De Vries P, Villalón CM, Saxena PR (1999) Pharmacological aspects of experimental headache models in relation to acute antimigraine therapy. Eur J Pharmacol 375(1-3):61-74. https://doi.org/10.1016/s00142999(99)00197-1

7. Goadsby PJ, Edvinsson L (1993) The trigeminovascular system and migraine: studies characterizing cerebrovascular and neuropeptide changes seen in humans and cats. Ann Neurol 33(1):48-56. https://doi.org/10.1002/ana. 410330109

8. Ashina M, Hansen JM, Do TP, Melo-Carrillo A, Burstein R, Moskowitz MA (2019) Migraine and the trigeminovascular system-40 years and counting. Lancet Neurol 18(8):795-804. https://doi.org/10.1016/S1474-4422(19)30185-1

9. Ornello R, Casalena A, Frattale I, Gabriele A, Affaitati G, Giamberardino MA et al (2020) Real-life data on the efficacy and safety of erenumab in the Abruzzo region, Central Italy. J Headache Pain. 21:32. https://doi.org/10. 1186/s10194-020-01102-9

10. Headache Classification Committee of the International Headache Society (IHS) (2018) The International Classification of Headache Disorders, 3rd edition. Cephalalgia 38:1-211. https://doi.org/10.1177/0333102417738202

11. Sarchielli P, Pini LA, Zanchin G, Alberti A, Maggioni F, Rossi C et al (2006) Clinical-biochemical correlates of migraine attacks in rizatriptan responders and non-responders. Cephalalgia. 26(3):257-265. https://doi.org/10.1111/j. 1468-2982.2005.01016.x

12. Goadsby PJ, Reuter U, Hallström $Y$, Broessner G, Bonner JH, Zhang F et al (2017) A controlled trial of Erenumab for episodic migraine. N Engl J Med 377(22):2123-2132. https://doi.org/10.1056/NEJMoa1705848

13. Faul F, Erdfelder E, Lang AG, Buchner A (2007) $G^{*}$ power 3: a flexible statistical power analysis program for the social, behavioral, and biomedical sciences. Behav Res Methods 39(2):175-191. https://doi.org/10.3758/ bf03193146

14. Barbanti P, Aurilia C, Egeo G, Fofi L (2019) Erenumab: from scientific evidence to clinical practice-the first Italian real-life data. Neurol Sci 40(Supp 1):177-179. https://doi.org/10.1007/s10072-019-03839-x

15. Lambru G, Hill B, Murphy M, Tylova I, Andreou AP (2020) A prospective realworld analysis of erenumab in refractory chronic migraine. J Headache Pain 21(1):61. https://doi.org/10.1186/s10194-020-01127-0

16. Russo A, Silvestro M, Scotto di Clemente F, Trojsi F, Bisecco A, Bonavita S et al (2020) Multidimensional assessment of the effects of erenumab in chronic migraine patients with previous unsuccessful preventive treatments: a comprehensive real-world experience. J Headache Pain 21:1-69. https:// doi.org/10.1186/s10194-020-01143-0

17. Raffaelli B, Kalantzis R, Mecklenburg J, Overeem LH, Neeb L, Gendolla A et al (2020) Erenumab in chronic migraine patients who previously failed five first-line Oral prophylactics and OnabotulinumtoxinA: a dual-center retrospective observational study. Front Neurol 11:417. https://doi.org/10. 3389/fneur.2020.00417
18. Kanaan S, Hettie G, Loder E, Burch R (2020) Real-world effectiveness and tolerability of erenumab: a retrospective cohort study. Cephalalgia 40(13): 1511-1522. https://doi.org/10.1177/0333102420946725

19. Matteo E, Favoni V, Pascazio A, Pensato U, Benini M, Asioli GM et al (2020) Erenumab in 159 high frequency and chronic migraine patients: real-life results from the Bologna headache center. Neurol Sci 41(Suppl 2):483-484. https://doi.org/10.1007/s10072-020-04667-0

20. Cheng S, Jenkins B, Limberg N, Hutton E (2020) Erenumab in chronic migraine: an Australian experience. Headache 60(10):2555-2562. https://doi. org/10.1111/head.13968

21. Tepper S, Ashina M, Reuter U, Brandes JL, Doležil D, Silberstein S et al (2017) Safety and efficacy of erenumab for preventive treatment of chronic migraine: a randomised, double-blind, placebo-controlled phase 2 trial. Lancet Neurol 16(6):425-434. https://doi.org/10.1016/S1474-4422(17)30083-2

22. Lovati C, Giani L, Mariotti D, Alessandro C, Tabaee Damavandi P, Mariani C, Pantoni $L$ (2018) May migraine attack response to triptans be a predictor of the efficacy of Onabotulinum toxin-a prophylaxis? Neurol Sci 39(Suppl 1): 153-154. https://doi.org/10.1007/s10072-018-3388-0

23. Eren OE, Gaul C, Peikert A, Gendolla A, Ruscheweyh R, Straube A (2020) Triptan efficacy does not predict onabotulinumtoxinA efficacy but improves with onabotulinumtoxinA response in chronic migraine patients. Sci Rep 10(1):11382. https://doi.org/10.1038/s41598-020-68149-1

24. Burstein R, Blumenfeld AM, Silberstein SD, Manack Adams A, Brin MF (2020) Mechanism of action of OnabotulinumtoxinA in chronic migraine: a narrative review. Headache 60(7):1259-1272. https://doi.org/10.1111/head. 13849

25. Burstein R, Zhang X, Levy D, Aoki KR, Brin MF (2014) Selective inhibition of meningeal nociceptors by botulinum neurotoxin type a: therapeutic implications for migraine and other pains. Cephalalgia. 34(11):853-869. https://doi.org/10.1177/0333102414527648

26. Melo-Carrillo A, Strassman AM, Nir RR, Schain AJ, Noseda R, Stratton J et al (2017) Fremanezumab-a humanized monoclonal anti-CGRP antibodyinhibits thinly Myelinated $(A \delta)$ but not unmyelinated $(C)$ meningeal Nociceptors. J Neurosci 37(44):10587-10596. https://doi.org/10.1523/ JNEUROSCI.2211-17.2017

27. Sacco S, Bendtsen L, Ashina M, Reuter U, Terwindt G, Mitsikostas DD et al (2019) European headache federation guideline on the use of monoclonal antibodies acting on the calcitonin gene related peptide or its receptor for migraine prevention. J Headache Pain 20(1):6. https://doi.org/10.1186/ s10194-018-0955-y

\section{Publisher's Note}

Springer Nature remains neutral with regard to jurisdictional claims in published maps and institutional affiliations.

Ready to submit your research? Choose BMC and benefit from:

- fast, convenient online submission

- thorough peer review by experienced researchers in your field

- rapid publication on acceptance

- support for research data, including large and complex data types

- gold Open Access which fosters wider collaboration and increased citations

- maximum visibility for your research: over $100 \mathrm{M}$ website views per year

At $\mathrm{BMC}$, research is always in progress.

Learn more biomedcentral.com/submissions 